\title{
Formalize the Software Quality Measurement for Heterogeneous Requirements
}

\author{
Edwin Mit \\ Faculty of Computer Science and Information \\ Universiti Malaysia Sarawak \\ 94300 Kota Samarahan, Sarawak. \\ edwin@fit.unimas.my
}

\author{
Cheah Wai Shiang \\ Faculty of Computer Science and Information \\ Universiti Malaysia Sarawak \\ 94300 Kota Samarahan, Sarawak. \\ cwshiang@fit.unimas.my
}

\begin{abstract}
There are two main challenges in measuring quality of software requirements: (i) There is no single model that can precisely represent the properties of heterogeneous requirements, and (ii) how to derive the quantitative measurement of the requirement properties that can be used to measure the software requirements quality? The issue of quality measurement is to define the benchmark or baseline used to transform the qualitative measurement to the quantitative measurement, and to date there are lack of particular rules or properties used to assess whether the propose software requirements fulfills the criteria. Therefore, this paper presents and discusses the framework of a new formal platform for assessing the quality of heterogeneous software requirements, which are obtain from open innovations. Hence, with this model the reliability of the software to be produced is known. This is an important study which, lead to the reduction of software failure in particular for safety-critical system
\end{abstract}

Keywords—software quality, formal methods, requirement measurement, open innovation

\section{INTRODUCTION}

Open innovation enables us innovate faster and continuously lowering the risk and expenses of bringing new products and services to market [1]. Agile methods, such as SCRUM and eXtreme Programming (XP) [2] has a similar goal, that is to bring the new product to the market faster. In the Agile approach, the interactive interaction between customers and developers is important in order to gain immediate feedback. Agile methods are in a way similar to open innovation, whereby customers are actively involved in the software development process, so that the development team can gather, compile and build open ideas in order to obtain the best solution. In order to increase the effectiveness of the customer on an eXtreme Programming (XP) project, patterns of roles and practices of customers has been defined in [3]. However, in Agile methods, their feedback and innovations are limited to the customers directly involved in the development project. C. Schmitt [4] had proposed the design of an Open Object Information Infrastructure to enable Open Innovation in the context of Ubiquitous Computing by enriching the semantics of virtual and physical object in the open innovation environment. In order to establish and maintain effective communication structures, a collection of patterns have been defined by C.Lescher [5], to help build a global team and to improve the effectiveness of communication and collaboration in global development projects. So far, there has been little discussion about the assessment of quality of the solutions from a variety of global talent sources. Recently there is a proposal by J. Mund [6] to investigate the possibilities and limitations of metrics in assessing the quality of (software) requirement specifications (SRS) and thereby aims to improve the understanding of the notion of quality for those specifications. Similar work has been carried out by Shahid Iqbal, [7] exploring how different metrics relating to different areas of software project, especially in requirement engineering. The focus of their research study is to evaluate and highlight the important of various performance metrics and propose additional metrics for requirement gathering and management and an effort to minimize the negative risk factors and improved adherence to quality assurance. In objectoriented approach, Martin Monperrus [8] define the requirements meta-model and use an automated measurement approach to specify requirements metrics. As software systems becoming more and more complex over time therefore software quality is also becoming major concern in software development [9]. For example, there are many service-oriented applications suffer from poor quality and are hard to evolve [10]. The quality problem is common problem in any development approaches as quality is measured entirely based on the requirement. The software components obtain from global talent such as open sources, being used and tested in different environment where the quality can be improved. However it lack of formal quantitative quality measurement to assess its quality metrics. Therefore, there is need for a more precise quality measurement model, which can formalize the software quality metrics as discuss in [6,7], so that the software requirement quality can be visualize in a quantitative manner. This is important for the developer to know earlier the quality of the software before it is being develop. And therefore can help developer improve quality metrics which may require high accuracy.

Therefore, this paper will present a new framework on how the quality of heterogeneous software requirements measurement is addressed in formal platform. The heterogeneous software requirements are the requirements that are captured from different domains. Section 2 will discussed the formulation of formal platform of the quality framework. Section 3 will discussed the formal platform used to measure the heterogeneous software requirements, and finally the conclusion and future work of this research. 